\title{
Transforming Health Care Delivery Through Consumer Engagement, Health Data Transparency, and Patient-Generated Health Information
}

\author{
D. Z. Sands $s^{a, b, d}$, J. S. Wald br,d, \\ - Beth Israel Deaconess Medical Center, Boston, MA, USA \\ ${ }^{b}$ Society for Participatory Medicine, Boston, MA, USA \\ ' RTI International, Waltham, MA, USA \\ ' Harvard Medical School, Boston, MA, USA
}

\begin{abstract}
Summary
Objective: Address current topics in consumer health informatics. Methods: Literature review.

Results: Current health care delivery systems need to be more effective in the management of chronic conditions as the popuIation turns older and experiences escalating chronic illness that threatens to consume more health care resources than countries can afford. Most health care systems are positioned poorly to accommodate this. Meanwhile, the availability of ever more powerful and cheaper information and communication technology, both for professionals and consumers, has raised the capacity to gather and process information, communicate more effectively, and monitor the quality of care processes.

Conclusion: Adapting health care systems to serve current and future needs requires new streams of data to enable better self-management, improve shared decision making, and provide more virtual care. Changes in reimbursement for health care services, increased adoption of relevant technologies, patient engagement, and calls for data transparency raise the importance of patient-generated health information, remote monitoring, non-visit based care, and other innovative care approaches that foster more frequent contact with patients and better management of chronic conditions.
\end{abstract}

\section{Keywords}

Chronic disease, consumer participation, delivery of health care, electronic health records, informatics

Yearb Med Inform 2014: 170-6

http://dx.doi.org/10.15265//Y-2014-0017

Published online August 15, 2014

\section{Introduction}

Much is written about patient engagement, collaborative team-based care, and meaningful physician-patient partnering to improve health outcomes, especially to address the growing burden of chronic disease. Approaching these ideals raises substantial information, communication, and technology challenges for both patients and providers on many levels. Advances in the use of electronic health records (EHRs) among providers, patient access to their health information, and health-focused mobile applications for information and tracking by patients offer potential avenues for improved engagement, collaboration, and team-based care. They also surface technical and non-technical challenges, including variation in the time, expertise, access to information, level of understanding, motivation, and technology environments that must be traversed when a health concern is being addressed.

The elevated promises and raised expectations of voluminous data streaming from multiple sources place a sharp focus on the patient, both as the major benefactor of big data, and as a significant data source through patient-generated health information (PGHI).

This paper explores opportunities and challenges that arise as tech-savvy consumers partner with technology-enabled providers to advance medical practice quality and care through an assessment of current medical practice and consumer technology use in that context, and a discussion of future implications.

\section{Current State of Ambulatory Medical Practice}

Medical care throughout much of the developed world suffers from inefficiency and ineffectiveness due to many factors including an over-dependence on episodic, visit-based care, poor patient engagement, communication and information discontinuity, and lack of effective use of information technology compared with other industries [1,2].

It is increasingly accepted that the effective use of an electronic health record improves record keeping, helps clinicians make better decisions, reduces errors, and helps to manage costs [3]. Through much of the developed world, adoption of the EHR has increased in recent years, although physicians in the United States and Canada remain laggards in adopting this technology [4]. In recent years, adoption has increased dramatically in the US, driven by financial incentives for "Meaningful Use" of "Certified EHR Technology" [5].

Even when physicians adopt EHRs, their actual use often falls short of the potential benefits, such as when decision support rules don't fully integrate test results and orders that would improve their precision, or when triggers 
are too broad, causing users to receive (and ignore) unnecessary alerts (and sometimes deactivate them). In one report from France, core functionality was found to be frequently missing from EHRs that are in common use [6]. Overall, frequent EHR and usability shortcomings effectively diminish the EHR's impact on cost-effectiveness or quality of care.

Health information exchange has significantly lagged behind EHR adoption in the U.S. $[4,7]$ and worldwide, limiting hoped-for electronic patient data flow between organizations and systems. Despite data standards to support health exchange and, in much of the world, universal health identifiers, progress has been slow. Some of the barriers have been a lack of sustainable business models, trust of health data acquired elsewhere, and mechanisms for importing third-party data into the EHR, or the development of a new virtual EHR model that leverages distributed data.

There has been progress in the U.S. As of April 2013, some $80 \%$ of all eligible hospitals and over $50 \%$ of all eligible providers received an incentive payment for meaningful use of EHRs [8]. EHR adoption has smoothed the way for integrated patient portal adoption [9]. Patient portals allow for secure patient-provider communication, facilitate patient education, enable online convenience transactions (e.g., prescription requests, appointments, referrals), and offer patients a view of their medical record data from the EHR and other systems. In certain settings, portals have been shown to reduce cost, improve quality, and improve the experience of care, although secure e-messaging along with other portal components have also been associated with higher utilization [10]. Although allowing patients to access their medical records and communicate with their health care providers electronically seems on its face to be good for patient engagement, there continues to be research literature controversy about its impact on health outcomes $[11,12]$. Data suggests a positive impact when accompanied by case management and other human interventions to improve patient engagement, since especially for chronic conditions, a combination of components is associated with benefits [13].

Physicians remain reluctant to adopt patient portals, but the success of these portals in a number of very large health systems in the U.S. has drawn a great deal of attention and has spawned robust studies. More importantly, the continuation of the Meaningful Use program (with financial incentives) in the U.S. is predicated on physicians exchanging e-messages with patients and providing them with the capability to view, download, and transmit information from their medical records [14].

Most portals that share patient records online restrict the information displayed to selected components. For example, while access to the medication list, problem list, visit history, and test results is common, the clinical encounter notes of EHRs have almost always been withheld. In 2010, a three-site study of progress notes online sharing with patients [15] showed that the majority of patients read their notes $(90 \%)$, felt more in control of their care, and reported greater medication adherence. The majority of doctors reported no negative impact of sharing notes with patients. Adopting the practice of sharing doctor's notes, the U.S. Veterans Administration Hospitals have reported high patient satisfaction [16]. Progress in expanding patient access to online notes and other chart information is anticipated to continue [17].

While providing patient access to their records is new for many physician practices and hospitals, some health care providers are offering entirely new convenience services. For example, in response to insufficient access to their usual source of care, "retail clinics" with evening and weekend hours are now proliferating, offered by pharmacy chains in the U.S. CVS Minute Clinic [18] and Walgreen's Healthcare Clinic [19] offer limited services provided by nurse practitioners for a modest cash payment and promise to send a visit summary to the primary care physician. Another new service connecting patients to an available physician by phone, online chat, or videoconference for basic health services is gaining traction in some areas [20]. The use of telemedicine to deliver care to underserved areas is also becoming more common as the cost and quality of the hardware has improved along with network bandwidth.

Remote monitoring using passive lowcost sensors and question-asking systems using mobile phones are now offered to some patients, who transmit their weight, blood pressure, medication adherence, and other parameters to their health care team to monitor treatment response. These remote monitoring programs are showing promise when used as part of a care management program such as to prevent re-hospitalization in heart failure patients or to improve home management of diabetes [21-25] through improved alerting and reduced barriers to communication and data sharing when appropriate.

\section{Consumer Trends}

\section{Consumers and Technology Use}

The U.S. consumer electronics industry, with almost $\$ 210$ billion in revenues in 2012, up $5 \%$ from the previous year, projects 116 million tablets, 130 million smartphones, and 26 million laptop/notebook computers will be sold [26] in 2013 with sales of mobile devices outnumbering PCs [27] in 2014 and mobile devices outnumbering humans on the planet [28]. The Internet is everywhere, whether experienced through a web browser on a laptop computer or "in my pocket" on a mobile (and increasingly, wearable) device.

Consumers spend nearly 8.25 hours per day, $50 \%$ of their waking time, with radio, television, or internet media. Over $85 \%$ of U.S. adults own a cell phone and 53\% of them own a smartphone that is within reach most of the time $[29,30]$. The time consumers spend on health concerns varies, but even for the patient with a chronic condition who has eight physician visits per year with associated laboratory and waiting time, it is tiny in comparison to other technology-mediated activities in which patients are engaged.

Most consumers who own multiple devices use more than one at a time, switching seamlessly between them throughout the day. For example, marketing studies of consumers show sequential and simultaneous (in parallel) use of different devices such as laptops, tablets, and phones to complete retail purchases, to self-notify progress during multi-step transactions, and to invoke search during a transaction [31]. Understanding and applying these findings is relevant to the 
effective design and adoption of consumer health-related technologies. Consumers owning multiple devices report that the situational context, the amount of time available, the goal they would like to accomplish, their location, and their attitude or state of mind drive their choice and sequence of devices during sequential usage.

Understanding the use of social networking sites may also be important for the design and use of technology for health. Over 50\% of the U.S. population are social network site users, with more than half between the ages of 12-34 years, with slightly more females (54\%) than males (46\%), and with the most rapid growth in the 65+ age group (50\% growth from 2011 to 2012, from 15\% to $23 \%$ ) [32]. Two-thirds of Facebook users access the site via mobile devices [33], and mobile users view the site almost 14 times per day [34]. The extensive use of social networking could be a big opportunity for addressing consumer's health concerns, although more research is needed [35].

\section{Consumer Demand for Health Care}

Consumers are expected to have greater interactions with health care services over the next few decades as the population gets older, experiences more chronic illness, gains insurance but with greater out-ofpocket costs, receives EHR-based care, and makes greater use of technologies for health.

The doubling of the over-age- 65 population to 89 million by $2050[36,37]$ disproportionately raises health service demands for conditions more prevalent with age. For example, significant growth in patients with Parkinson's disease ( $68 \%$ by 2030), Alzheimer's disease $(40 \%$ by 2025$)$, cancer $(30 \%$ by 2020 ), diabetes ( $21 \%$ by 2025$)$, and stroke or cardiovascular disease $(27 \%$ by 2025$)$ are predicted by public health models, compared with prevalence in recent years [38].

Expanded health insurance coverage in the U.S. under the Affordable Care Act could increase the number of insured from $82 \%$ to $92 \%$ nationwide [38], while accelerated rollout of electronic health records in the U.S. means more patients are experiencing EHR-based care. Together, these shifts may have mixed effects. For example, growth in patients with diabetes is likely to increase demand for health services and physicians, especially if mid-level providers serve additional patients or if there's growth in remote monitoring and pre-visit intervention for chronic condition patients. The future impact of consumer Internet-based information seeking, health tracking, and electronic information sharing is uncertain, and requires further study.

\section{Consumer Technology for Health}

Pew Internet and American Life data shows that among the $45 \%$ of U.S. adults who live with one or more chronic condition, $72 \%$ of them use the Internet and are more likely (compared to those without a chronic condition) to gather online information, consult online reviews, and read or watch something online about someone else's personal health experience [39] than others without a chronic condition who were surveyed. Those with a chronic condition are also more likely to check with a physician concerning what they find online, with about half reporting that what they found online was confirmed by a physician, at least in part, and twenty percent reporting receiving a different opinion than what they found online.

Pew data [40] also finds that $69 \%$ of U.S. adults keep track of at least one health indicator such as weight, diet, exercise routine, or a symptom for themselves or others. Diet, weight or exercise $(60 \%)$ were the most common, followed by any other health indicators like blood pressure, sleep patterns, or headaches $(33 \%)$. Those with a chronic condition were no more likely to track diet/ weight/exercise, but were more likely to track "other health indicators" if they had one chronic condition ( $40 \%$ trackers vs. $33 \%$ overall) or multiple chronic conditions $(62 \%$ trackers). The majority of trackers "keep it in their head" (49\%), with others keeping tracking data in a notebook (34\%) or using some form of technology (21\%).

Patient-generated health information has received increasing focus in recent years, including an ONC-sponsored white paper [41] and report from a technical expert panel [42]. These data are "health-related data - including health history, symptoms, biometric data, treatment history, lifestyle choices, and other information-created, recorded, gathered, or inferred by or from patients or their designees (i.e., their care partners or those who assist them) to help address a health concern." The challenges of obtaining and using patient-generated health information (PGHI) to improve patient-centered care include technical issues (e.g. capturing, transmitting, and reviewing electronic data), policy issues (e.g. who is accountable for each step in the handling of this information), and practical issues (e.g. what thresholds are useful for timely review of received information, and for automated alerting for out-of-range information). The Pew tracking data underscores the opportunity for impact. Only some trackers share their information with their physician (18\%) or someone else $(16 \%)$ even though half of them (46\%) update their tracking data on a regular basis. Higher levels of sharing and updates occur among trackers living with $2+$ chronic conditions, who say tracking has affected their overall approach to maintaining health $(56 \%)$, led them to ask a doctor new questions or seek a second opinion (53\%), or affected a decision about how to treat an illness or condition (45\%). The potential for tracking to be an aid in communication, understanding, and making health decisions is clearly growing.

Expanding technology use alone does not guarantee that making health decisions and managing health concerns will be easier. And while research on activated patients such as trackers is informative, understanding the process of conversion from an uninvolved to highly engaged patient is critical. Patients often experience physical, mental, or emotional stress, time urgency, poor access to key resources, and difficulty in understanding due to limited health literacy, work or family pressures, financial distress, or simply a lack of motivation [43]. When studied ethnographically, online experiences may help patients develop and test their understanding of health concerns they face, compare their experience with others, and decide when to challenge or trust professional authority as they work with physicians [44]. More research is needed in the use of social media in health communications to demonstrate its potential benefits and explore 
Transforming Health Care Delivery Through Consumer Engagement, Health Data Transparency, and Patient-GeneratedHealth Information

concerns such as information quality and personal disclosures [35]. Fully engaging as a patient, especially one with a chronic condition that requires routine (if not daily) attention, is a growing challenge with important technological and non-technological dimensions [45].

\section{Discussion}

Over the past century, as medical science has eliminated or cured an astonishing array of acute and infectious diseases, the burden of chronic disease has grown. Chronic care models emphasize more continuous care, greater patient engagement, and closer monitoring of data [25, 45-47], creating a mismatch between the acute episodic care model favored by most health care delivery systems, and a population that increasingly requires more longitudinal care for chronic illness. Care coordination requirements are high. For example, in the U.S. people age 65 and above see an average of seven different physicians per year [48], placing a huge communication and data exchange burden on both the patients and their health care providers. Quality patient care, especially for chronic conditions, depends upon care coordination and information management.

Important health decisions are made by patients and caregivers away from professional health care, considered "off the map" [49] from the perspective of health care professionals, or the "care between the care," [50] and many patient behaviors profoundly impact health outcomes [51]. Moreover, health care providers often underestimate the substantial work involved in being a patient [52]. Failure to incorporate activities, information, and decisions made away from the doctor's office may have undesired consequences for patient health.

For example, over $20 \%$ of patients have a higher blood pressure in the medical office than they actually have at home [53], while other patients who are normotensive in the office may be hypertensive at home [54]. False positives in the medical office can subject patients to costly and potentially harmful management that is not needed, and false negatives can be even more harmful since hypertension may go untreated. Home ambulatory blood pressure monitoring can help, especially if significant results are communicated before the patient's next scheduled visit to avoid treatment delays. In Sweden at the Karolinska University Hospital, many rheumatology practices collect patient-reported information that is automatically analyzed, trended, and summarized for use by the patient (in self-care), the provider (during a visit), and researchers (for public health reporting and regional/national research data aggregation) [55].

Provider use of information collected from patients and caregivers in their "daily routines" outside the clinical setting and concerning not just their health activities, but their values and preferences, is not new. But the importance of improving many decisions currently made without this information is increasingly being recognized $[56,57]$. Technologies and practices that facilitate collection and sharing of patient-generated health information [41] range from data from monitors for weight, blood pressure, glucose, activity, or falls, to psychometric data entry on smart phones, to patient preference information useful for shared decision-making [42].

The great challenge in having additional information is that its value reflects both the resources required to obtain and process the information, and the benefits actually realized from its use. Methods to predict and capture novel information that makes a difference in health outcomes or management are critical. Changing the behavior of patients, caregivers, and providers to acquire and incorporate these new streams of information will require education, changes in workflow, development and adaptation of technology, realignment of incentives, policy changes such as Meaningful Use Stage 3 in the U.S., and addressing potential physician liability.

As partners in care, not only can providers benefit from PGHI shared by the patient, but patients can benefit from access to their own medical record information shared by the provider. This parity of information access, part of the principle of "nothing about me without me" [58], is important to effective engagement [47]. As systems enabling full transparency of patient information for-the-provider, and provider information for-the-patient are deployed, new challenges will naturally emerge since shared information alone does not ensure benefit. The U.S. meaningful use regulations are intended to increase dramatically the sharing of EHR information with patients [14].

Reimagining what is optimal for chronic care management involves informed and activated patients, a prepared, proactive clinical team, and care that transcends the boundaries between the community and the health care system [46] (see Figure 1).

Some of this is supported by the adoption of technologies as described above. But some requires reconfiguring medical practices so that care is delivered as a team, with the patient as an essential participant, and each member practicing to their highest level of training or expertise.

Fee-for-service reimbursement drives an episodic focus of health care delivery, which is appropriate for some forms of care, but provides a disincentive to manage care longitudinally or to focus on long-term quality and prevention goals. Healthcare payment reform is beyond the scope of this paper, but as governments and other payers evolve their payment models to strengthen payment for quality (with rewards for both quality of care and patient satisfaction), sharing in cost savings if quality targets are achieved, case management payments, and additional reimbursement for all-encompassing care, the incentives for providing more continuous care using more "daily routine" data may shift. In the U.S., formation of "accountable care organizations" (ACOs) and practices serving as "Patient-Centered Medical Homes" exemplify this shift [56, 57]. Given what large corporations such as Google, Facebook, Amazon, and large retailers understand about their customers by routinely compiling data about their activities and preferences in real-time, there may be non-healthcare industry models for improving the patient experience, outcomes, and satisfaction that inform successful practices for the collaborative care of patients with chronic conditions.

Given the anticipated growth in patient-generated and provider-generated 


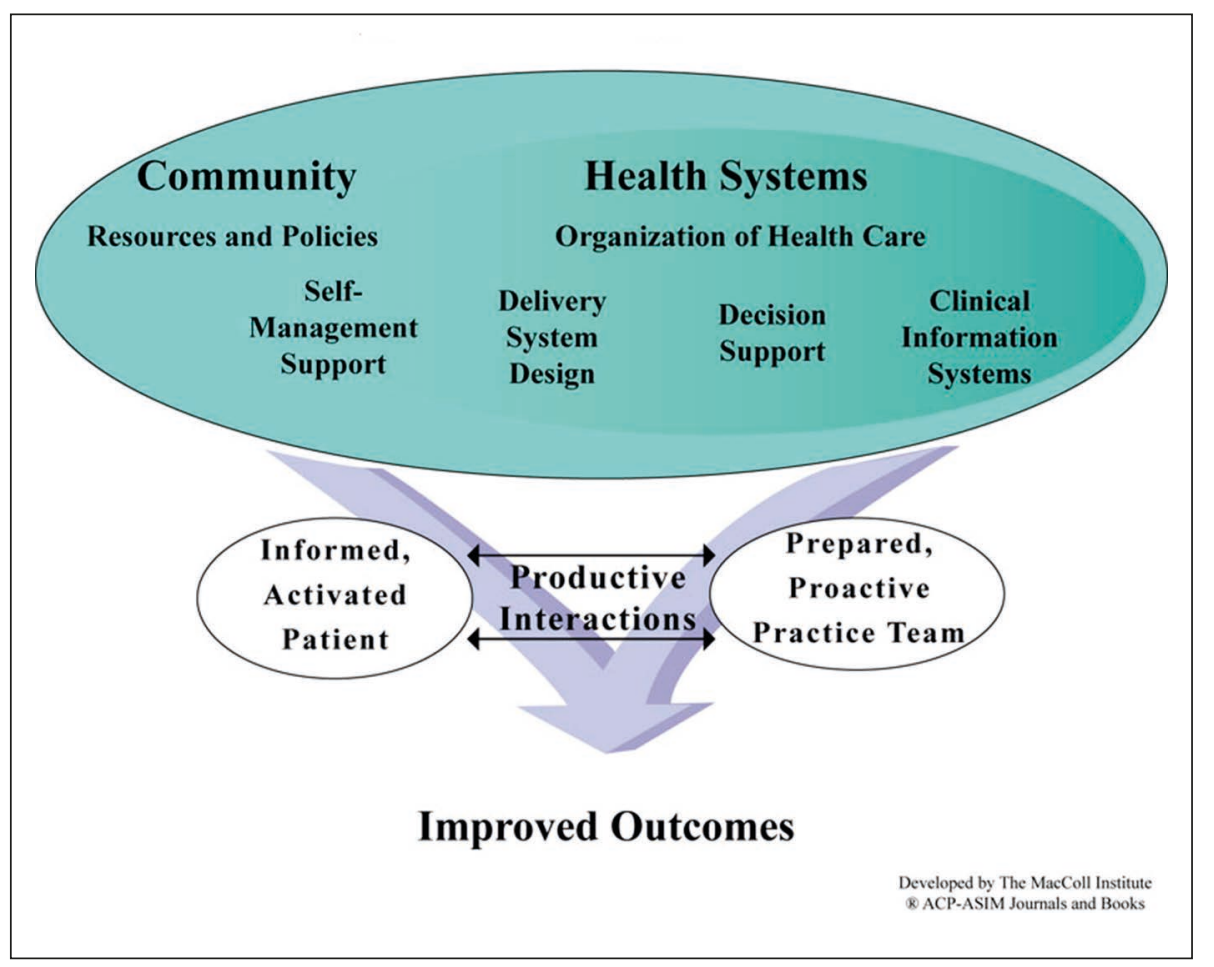

Fig. 1 The Care Model. Copyright 1996-2014 The MacColl Center. The Improving Chronic Illness Care program is supported by The Robert Wood Johnson Foundation, with direction and technical assistance provided by Group Health's MacColl Center for Health Care Innovation.

health information as EHR use becomes more widespread and payment models shift, there are a number of informatics and research challenges that should be addressed.

\section{Standards}

Standards that support representation and secure transmission of patient-generated data and EHR-generated data, which exist in areas such as clinical conditions [59], observations [60], medical procedures [61], pharmaceuticals [62], and medical documents [63], will need to expand to include consumer medical device data, patient-reported outcomes, and a wide range of information types and use cases.

While standards are important for interoperability, they may also constrain information flow or inadvertently limit or alter its context. Standards should be developed and applied with care, especially when information technology is used in the service of communication rather than computation [64].

\section{Consumer Vocabularies, Interfaces, and Workflows}

Consumers need easily understood health information and usable devices that support their workflow. Several challenging areas need further work: a) translating problem list vocabularies and other jargon-rich medical documentation into health information that is more easily understood by consumers [65], b) using universal design principles to improve consumer applications and make devices usable by patients who may have limited literacy, and c) understanding how and when patients use technology to track their health information.

\section{Data Provenance and Integrity}

Incorporating patient data into the electronic health record from outside sources such as other medical facilities, caregivers and patients, or third parties (e.g. commercial genomic data) requires reliable and trusted mechanisms for identifying and acquiring unadulterated, time-stamped data from known sources. This process also requires that ambiguous health identifiers (with or without a universal health identifier) be avoided, an important area of further study.

\section{Acceptance of PGHI into the Electronic Health Record}

Further work is needed in developing the technical and procedural mechanisms for identifying and processing PGHI submitted to a provider, and policies guiding review and documentation of relevant information in a consistent way across organizations. This includes the need for intelligent filtering, trending, and alerting algorithms to evaluate potentially large streams of patient phenomic and genomic data when appropriate. While concerns about the "onslaught of PGHI" that may consume the time of providers are not uncommon [42], providers already have formal and informal policies that guide their practice of accepting and reviewing information offered by patients and caregivers. A sociotechnical approach to designing the use of PGHI will appropriately and usefully balance the emphasis on both the human dimension of the work and the technology [66].

\section{Patient Participation and Clinical Impact of PGHI}

The value of expanding the technical and procedural capabilities for sharing PGHI, incorporating it into decision-making, documenting it, and engaging patients, caregivers, and providers effectively needs greater exploration through formal and applied research, as does patient acceptability of these technologies.

As financial incentives and bidirectional information flows between patients and providers become more common, we will inevitably learn a great deal about how to use technology and information more effectively. These changes to health care systems will lead to greater creativity and innovation among health care providers, who will find new ways of delivering care in ways that are more information-driven, engaging, cost-effective, and efficient, and better suited to 
Transforming Health Care Delivery Through Consumer Engagement, Health Data Transparency, and Patient-GeneratedHealth Information

meeting the needs of all patients, especially those with chronic diseases. In addition, patients and caregivers will no longer be limited in their level of engagement in care activities and care teams.

\section{Conclusion}

Visit-based care inefficiencies, payment model disincentives, workflow challenges, time limitations, and technical constraints add to the information challenges of health care that affect providers, patients, caregivers, and health organizations, making it especially difficult to manage the increasing burden of chronic disease. Rapidly advancing consumer technologies that leverage mobile sensors, geo-location tagging, personal profiles, and streaming data illustrate how information technology is leveraged in many industries. Patient-generated health information, enabled by data transparency and consumer engagement, is not a panacea, but can help address information gaps in important areas, leverage untapped patient experience, and offer information that will improve self-management, provider-directed, and joint decisions made by patients and providers together and facilitate more frequent contacts with patients for better management of chronic conditions.

\section{References}

1. Blumenthal D. Wiring the health system—origins and provisions of a new federal program. N Engl J Med 2011;365(24):2323-9.

2. Pozin I. Industry to watch in 2014: Healthcare tech. 2013. Available at: http://www.forbes.com/ sites/ilyapozin/2013/10/17/industry-to-watch-in2014-healthcare-tech/

3. Hillestad R, Bigelow J, Bower A, Girosi F, Meili $\mathrm{R}$, Scoville R, et al. Can electronic medical record systems transform health care? Potential health benefits, savings, and costs. Health Aff (Millwood) 2005;24(5):1103-17.

4. Jha AK, Doolan D, Grandt D, Scott T, Bates $\mathrm{DW}$. The use of health information technology in seven nations. Int J Med Inf 2008;77(12):848-54.

5. Blumenthal D, Tavenner M. The "meaningful use" regulation for electronic health records. $\mathrm{N}$ Engl J Med 2010;363(6):501-4.

6. Darmon D, Sauvant R, Staccini P, Letrilliart L. Which functionalities are available in the electronic health record systems used by french general prac- titioners? An assessment study of 15 systems. Int J Med Inf 2014;83(1):37-46.

7. Blumenthal D. Implementation of the federal health information technology initiative. N Engl J Med 2011;365(25):2426-31.

8. HHS. Doctors and hospitals' use of health it more than doubles since 2012. 2013. Available at: http://www.hhs.gov/news/ press/2013pres/05/20130522a.html. Accessed January 9.

9. Tang PC, Ash JS, Bates DW, Overhage JM, Sands DZ. Personal health records: Definitions, benefits, and strategies for overcoming barriers to adoption. J Am Med Inform Assoc 2006;13(2):121-6.

10. Palen TE, Ross C, Powers JD, Xu S. Association of online patient access to clinicians and medical records with use of clinical servicesonline patient access and use of clinical services. JAMA 2012;308(19).

11. Goldzweig CL, Orshansky G, Paige NM, Towfigh AA, Haggstrom DA, Miake-Lye I, et al. Electronic patient portals: Evidence on health outcomes, satisfaction, efficiency, and attitudes. Ann Intern Med 2013;159(10).

12. Wagner PJ, Dias J, Howard S, Kintziger KW, Hudson MF, Seol YH, et al. Personal health records and hypertension control: A randomized trial. J Am Med Inform Assoc 2012;19(4):626-34.

13. Stellefson M, Dipnarine K, Stopka C. The chronic care model and diabetes management in us primary care settings: A systematic review. Preventing chronic disease 2013 Feb;10:E26. PMID: 23428085.

14. CMS. Eligible professional's guide to stage 2 of the ehr incentive programs. 2013. Available at: http:// www.cms.gov/Regulations-and-Guidance/Legislation/EHRIncentivePrograms/ Downloads/Stage2_Guide_EPs_9_23_13. pdf. Accessed January 9 .

15. Delbanco T, Walker J, Bell SK, Darer JD, Elmore JG, Farag N, et al. Inviting patients to read their doctors' notes: A quasi-experimental study and a look ahead. Ann Intern Med 2012;157(7):461-70.

16. Woods SS, Schwartz E, Tuepker A, Press NA, Nazi KM, Turvey CL, et al. Patient experiences with full electronic access to health records and clinical notes through the my healthevet personal health record pilot: Qualitative study. J Med Internet Rese 2013;15(3).

17. OpenNotes. Myopennotes website. 2013 Available at: http://www.myopennotes.org. Accessed January 9.

18. CVS minute clinic. Available at: http://www. minuteclinic.com.

19. Walgreens healthcare clinic. Available at: http:// www.walgreens.com/topic/pharmacy/ healthcare-clinic.jsp.

20. American Well. Available at: http://www.americanwell.com.

21. Quinn CC, Shardell MD, Terrin ML, et al. Cluster-randomized trial of a mobile phone personalized behavioral intervention for blood glucose control. Diabetes Care 2011;34(9):1934-42.

22. Paré G, Moqadem K, Pineau G, St-Hilaire C. Clinical effects of home telemonitoring in the context of diabetes, asthma, heart failure and hypertension: A systematic review. J Med Internet Res 2010;12(2).

23. Darkins A, Ryan P, Kobb R, Foster L, Edmonson E, Wakefield B, et al. Care coordination/ home telehealth: The systematic implementation of health informatics, home telehealth, and disease management to support the care of veteran patients with chronic conditions. Telemed J E Health 2008;14(10):1118-26.

24. Shea S, Weinstock RS, Teresi JA, Palmas W, Starren J, Cimino JJ, et al. A randomized trial comparing telemedicine case management with usual care in older, ethnically diverse, medically underserved patients with diabetes mellitus: 5 year results of the ideatel study. JAm Med Inform Assoc 2009;16(4):446-56.

25. Pandor A, Gomersall T, Stevens JW, Wang J, Al-Mohammad A, Bakhai A, et al. Remote monitoring after recent hospital discharge in patients with heart failure: A systematic review and network meta-analysis. Heart 2013 Dec;99(23):1717-26.

26. Anon. Consumer electronics industry revenues to reach record-high $\$ 209$ billion in 2013, according to cea. 2013. Available at: http://www. ce.org/News/News-Releases/Press-Releases/2013-Press-Releases/CE-Industry-Revenues-to-Reach-Record-High-\$209-Bil. aspx. Accessed January 9.

27. Evans B. What does mobile scale mean? (weblog). 2013. Available at: http://ben-evans.com/benedictevans/2013/12/18/what-does-mobilescale-mean. Accessed January 9.

28. Cisco. White paper: Visual networking index: Global mobile data traffic forecast update, 2012-2017. 2013. Available at: http://www.cisco. $\mathrm{com} / \mathrm{c} / \mathrm{en} / \mathrm{us} /$ solutions/collateral/service-provider/ visual-networking-index-vni/white_paper_c11520862.html

29. Fox S, Duggan M. Mobile health 2012. Pew Internet and American Life Project 2012;8.

30. Edison Arbitron Research. The infinite dial 2013: Navigating digital platforms. 2013. Available at: http://www.edisonresearch.com/wp-content/uploads/2013/04/Edison_Research_ Arbitron_Infinite_Dial_2013.pdf.

31. Google. The new multi-screen world. 2012. Available at: http://www.google.com/think/ research-studies/the-new-multi-screenworld-study.html. Accessed January 9.

32. Anon. The social habit. Edison/Arbitron Internet and Multimedia Research Series 2012.

33. Facebook. Investor relations quarterly financial report jan-mar 2014. 2014. Available at: http:// investor.fb.com/releasedetail.cfm?ReleaseID $=842071$.

34. IDC research report - Always connected: How smartphones and social keep us engaged; 2013.

35. Moorhead SA, Hazlett DE, Harrison L, Carroll JK, Irwin A, Hoving C. A new dimension of health care: Systematic review of the uses, benefits, and limitations of social media for health communication. J Med Internet Res 2013;15(4).

36. Jacobsen LA, Kent M, Lee M, Mather M. America's aging population: Population Reference Bureau; 2011. Available at: http://www.prb.org/ pdf11/aging-in-america.pdf 
37. Vincent GK, Velkoff VA. The next four decades: The older population in the united states: 2010 to 2050: US Department of Commerce, Economics and Statistics Administration, US Census Bureau; 2010.

38. Dall TM, Gallo PD, Chakrabarti R, West T, Semilla AP, Storm MV. An aging population and growing disease burden will require alarge and specialized health care workforce by 2025 . Health Aff (Millwood) 2013;32(11).

39. Fox S, Duggan M. The diagnosis difference. Pew Internet and American Life Project 2013. Available at: http:/www.pewinternet.org/2013/11/26/ the-diagnosis-difference/

40. Fox S, Duggan M. Tracking for health. Washington, DC: Pew Internet \& American Life Project, January http://www pewinternet org/Reports/2013/Tracking-for-Health aspx 2013.

41. Shapiro M, Johnston D, Wald J, Mon D. Patient-generated health data. Office of the National Coordinator for Health Information Technology. Research Triangle Park, NC: RTI International; 2012.

42. Berry K. Final report of the technical expert panel on patient-generated health information; 2013.

43. O'Kane M, Buto K, Alteras T, Baicker K, Fifield $\mathrm{J}$, Griffin R, et al. Demanding value from our health care: Motivating patient action to reduce waste in health care. 2012.

44. Kivits J. Researching the'informed patient. Information, Communication \& Society 2004;7(4):510-30.

45. Clancy CM. Patient engagement in health care. Health Serv Res 2011;46(2):389-93.

46. Wagner EH. Chronic disease management: What will it take to improve care for chronic illness? Eff Clin Pract 1998 Aug-Sep;1(1):2-4. PMID: 10345255

47. Oldenburg J. Engage! Transforming healthcare through digital patient engagement. Chicago, IL: Health Information Management Systems Society
(HIMSS); 2013.

48. Pham HH, Schrag D, O'Malley AS, et al. Care patterns in medicare and their implications for pay for performance. NEngl J Med 2007;356(11):1130-9.

49. Ferguson T. Consumer health informatics. Healthc Forum J 1995 1995;38(1).

50. Brennan P, Swanberg W. Listening in the moment. interactions 2013;20(5):22-5.

51. Ford ES, Bergmann MM, Kroger J, Schienkiewitz A, Weikert C, Boeing H. Healthy living is the best revenge: Findings from the european prospective investigation into cancer and nutrition-potsdam study. Arch Intern Med 2009;169(15):1355.

52. Unruh KT, Pratt W. The invisible work of being a patient and implications for health care:" [the doctor is] my business partner in the most important business in my life, staying alive". Ethnographic Praxis in Industry Conference Proceedings; 2008: Wiley Online Library; 2008. p. 40-50.

53. Pickering TG, James GD, Boddie C, Harshfield GA, Blank S, Laragh JH. How common is white coat hypertension? JAMA1988;259(2):225-8.

54. Ohkubo T, Kikuya M, Metoki H, Asayama K, Obara T, Hashimoto J, et al. Prognosis of "masked" hypertension and "white-coat" hypertension detected by 24-h ambulatory blood pressure monitoring10-year follow-up from the ohasama study. J Am Coll Cardiol 2005;46(3):508-15.

55. Nelson EC, Hvitfeldt HF, Reid R, Grossman D, Lindblad S, Mastanduno, MP, et al. Using patient-reported information to improve health outcomes and health care value: Case studies from dartmouth, karolinska and group health: Technical Report. The Dartmouth Institute for Health Policy and Clinical Practice; 2012.

56. Rittenhouse DR, Shortell SM. The patient-centered medical home. JAMA 2009;301(19):2038-40.

57. Friedberg MW, Van Busum K, Wexler R, Bowen $\mathrm{M}$, Schneider EC. A demonstration of shared decision making in primary care highlights barriers to adoption and potential remedies. Health Aff (Millwood) 2013;32(2):268-75.

58. Delbanco T, Berwick DM, Boufford JI, Edgman-Levitan S, Ollenschläger G, Plamping $\mathrm{D}$, et al. Healthcare in a land called peoplepower: Nothing about me without me. Health Expect 2001;4(3):144-50.

59. IHTSDO. Snomed ct. Available at: http://www. ihtsdo.org/snomed-ct/.

60. LOINC. Loinc website. Available at: http:// loinc.org/.

61. AMA. Cpt codes. Available at: http://www. ama-assn.org/ama/pub/physician-resources/solutions-managing-your-practice/ coding-billing-insurance/cpt.page

62. UMLS. Nddf. Available at: http://www.nlm. nih.gov/research/umls/sourcereleasedocs/ current/NDDF/.

63. CDC. Clinical document architecture. Available at: http://www.cdc.gov/nhsn/CDA/index.html.

64. Coiera E. Stasis and adaptation. Stud Health Technol Inform 2012;194:11-9.

65. Zeng QZ. Consumer health vocabulary initiative. 2013. Available at: http://consumerhealthvocab.org/index.html?http $\% 3 \mathrm{~A} / /$ consumerhealthvocab.org/bibliography.html. Accessed 04/27/2014.

66. Coiera E. Four rules for the reinvention of health care. BMJ 2004;328(7449):1197.

Correspondence to:

Daniel Z. Sands, MD, MPH

56 Solon St., Suite 200

Newton, MA 02461, USA

Tel: +16172564775

Fax: + 16176636321

E-mail: Danny@DrDannySands.com 